\title{
La notion de limnicité est-elle suffisante pour caractériser l'empreinte spatiale et sociétale des plans d'eau?
}

Is the limnicity's concept really adequate to be characteristic of social and societal mark of water bodies?

Reicht der Begriff des Gewässeranteils zur Beschreibung der räumlichen wie gesellschaftlichen Auswirkungen von Gewässern aus?

Pascal Bartout and Laurent Touchart

\section{OpenEdition}

\section{Journals}

Electronic version

URL: http://journals.openedition.org/rge/5766

DOI: $10.4000 /$ rge.5766

ISSN: $2108-6478$

Publisher

Association des géographes de l'Est

Printed version

Date of publication: 22 April 2016

ISSN: 0035-3213

\section{Electronic reference}

Pascal Bartout and Laurent Touchart, «La notion de limnicité est-elle suffisante pour caractériser l'empreinte spatiale et sociétale des plans d'eau ? », Revue Géographique de l'Est [Online], vol.56 / n $1-2$ | 2016, Online since 03 July 2016, connection on 10 December 2020. URL : http:// journals.openedition.org/rge/5766; DOI : https://doi.org/10.4000/rge.5766

This text was automatically generated on 10 December 2020.

Tous droits réservés 


\title{
La notion de limnicité est-elle suffisante pour caractériser l'empreinte spatiale et sociétale des plans d'eau?
}

\author{
Is the limnicity's concept really adequate to be characteristic of social and \\ societal mark of water bodies? \\ Reicht der Begriff des Gewässeranteils zur Beschreibung der räumlichen wie \\ gesellschaftlichen Auswirkungen von Gewässern aus?
}

Pascal Bartout and Laurent Touchart

\section{Introduction}

1 Les travaux limnologiques à travers le monde ont de tout temps fortement privilégié les «lacs» naturels. Les indicateurs utilisés pour de telles études sont ainsi parfaitement adaptés à ce type de plans d'eau, de même que les typologies et régionalisations qui en découlent. Parmi les indices employés habituellement, la limnicité, qui signifie la part en eau lacustre naturelle d'un territoire, matérialise l'empreinte surfacique des lacs et se trouve être le seul indicateur spatial à même de permettre de comparer des territoires entre eux.

Bien que discutée par les différentes écoles et courants limnologiques, la limnicité est un marqueur international du fait lacustre progressivement élargi aux réservoirs artificiels. Pourtant, cet indicateur est aujourd'hui remis en question en raison de deux problématiques sans liens apparents l'une avec l'autre. D'une part, nombre de chercheurs ciblent depuis quelques années les petits plans d'eau dans leurs études (moins de 100 ha et même moins d'1 ha) afin de comprendre le rôle joué par ces petites masses d'eau dormantes dans le cycle du carbone par l'intermédiaire de leurs sédiments (Downing et al., 2008). D'autre part, les polices de l'eau s'adaptent aux 
contextes géographiques des territoires qu'elles doivent gérer : progressivement, les faits culturels complètent ceux naturels. Ceci se traduit dans le droit, notamment européen, par la présence du terme «plan d'eau » et non celui de «lac» dans la DCE-2000 (Directive Cadre Européenne sur l'Eau).

Or, si les textes évoluent, les études sur les différents types de plans d'eau manquent, et de fait, le décalage entre les objectifs fixés et les réalités de terrain s'accentue. Ainsi, à l'échelle de la France, les masses d'eau "plans d'eau» des six agences de l'eau reconnaissent uniquement 456 entités, alors que nous avons pu mettre en évidence plus de 554000 plans d'eau de plus d'un are (Bartout \& Touchart, 2013) : ainsi, le choix effectué par les agences de l'eau, autant scientifique que budgétaire, de ne cibler comme "plans d'eau » que les lacs naturels et les MEFM (masses d'eau fortement modifiées) de plus de 50 hectares entraîne la négation de l'existence de 99,92 \% des plans d'eau français et leur classement par défaut comme masses d'eau « cours d'eau ».

4 Ainsi, la très grande majorité des propriétaires et gestionnaires de plans d'eau en France, qui se pensent et se disent aménager des étangs, des mares ou des retenues collinaires, ne comprennent pas les exigences demandées par les différentes administrations en charge de la police de l'eau.

5 Pour permettre une meilleure évaluation des politiques de l'eau et leur efficience, il est donc important d'envisager toute la pluralité limnique. Le critère de la limnicité est-il alors suffisant pour permettre cette compréhension spatiale de la diversité naturelle et culturelle des milieux stagnants?

6 Pour répondre à cette question, nous envisagerons dans un premier temps l'étude de la limnicité et tout ce qu'elle sous-entend comme chiffres, méthodes et logiques scientifiques en lien avec la genèse de ce néologisme russe. Dans une seconde partie, nous nous interrogerons sur la pertinence spatiale de ce terme en France en lien avec les avancées récentes procurées par l'inventaire général des plans d'eau en France (Bartout \& Touchart, 2013). Enfin, l'approche systématique des plans d'eau par leur dimension surfacique sera débattue en lien avec les problématiques lentiques actuelles.

\section{La notion de « limnicité » ou comment caractériser l'empreinte spatiale des lacs?}

7 Les lacs, systèmes lentiques les plus imposants de la planète (la majeure partie des classifications internationales établissent la limite surfacique inférieure d'un lac à 100 ha selon Trëšnikov, 1988), ont suscité des travaux depuis fort longtemps, que ce soit à l'échelle nationale ou internationale. Pour caractériser leur empreinte spatiale, les scientifiques russes, à la suite des premières tentatives allemandes d'il y a un siècle, ont créé le néologisme «limnicité », terme qui sera ensuite introduit en France par M. Meybeck au milieu des années 1990.

\section{A. Genèse de ce terme.}

8 A la fin du XIX ème siècle, dans la continuité des avancées scientifiques en géomorphologie, potamologie et limnologie, les premiers décomptes de lacs naturels ont été réalisés à deux échelles distinctes :

9 - dans des régions bien circonscrites comme les lacs alpins par A. Delebecque (1898); 

plutôt la superficie cumulée des grands lacs. Pour L. Touchart (2014), «de ce fait, la surface en eau totale, $y$ compris en proportion de celle des continents, conduisant au concept de limnicité, était mieux connue que le nombre absolu de plans d'eau et leur densité par unité spatiale ». A. Penck nomma ce concept «part des lacs à la surface» que W. Halbfass quantifia à 2,5 millions de $\mathrm{km}^{2}$, soit 1,68 \% des terres émergées. Ces chiffres furent repris par l'ensemble de la communauté scientifique internationale jusqu'aux années 1960, voire plus tard encore dans le cas de certains manuels de limnologie (Wetzel, 2001). La principale avancée scientifique de la deuxième moitié du XX ${ }^{\mathrm{ème}}$ siècle provint de la prise en compte des plans d'eau de petite taille: elle est à mettre au crédit de l'école russe de limnologie. Appliqués au territoire soviétique, les résultats furent extrapolés à la planète : G.P. Tamrazyan (1974) fixa ainsi « la surface lacustre planétaire à 2,7 millions de $\mathrm{km}^{2}$, soit une limnicité de 1,81\%» (Touchart, 2014), chiffre confirmé par les travaux ultérieurs de C.E. Herdendorf $(1982,1990)$, M. Meybeck (1995a et b) ou S. Rianjine (2005) avec une marge d'erreur n'excédant pas 3 à $4 \%$.

12 Apporté en France par M. Meybeck en 1995 qui francise le terme russe " oziornost ${ }^{1}$ »en « limnicité » (« limnic ratio » en anglais), ce concept rencontre un vif succès mais se heurte à deux écueils méthodologiques importants que l'on retrouve dans la constitution du panel des masses d'eau «plans d'eau » par les Agences de l'eau : quelle limite inférieure doit-on donner au terme de « lac » et doit-on désormais comptabiliser les plans d'eau non naturels dont le nombre a explosé depuis les premières estimations des chercheurs allemands?

\section{B. Les limites du concept de limnicité.}

13 Surfaciquement, un consensus semble s'être opéré au niveau mondial fixant le seuil minimal du lac à $1 \mathrm{~km}^{2}$, soit 100 ha (Trëšnikov, 1988, Brochu et Michel, 1994), sans que cette décision soit le fruit "d'une réflexion géographique naturaliste sur le seuil à partir duquel un plan d'eau fonctionne comme un ensemble différencié en zones" (Touchart, 2014). C'est le seuil retenu par M. Meybeck quand il avance une limnicité de 0,09\% en France, soit à peine $5 \%$ de la limnicité moyenne mondiale fixée à $1,81 \%$. Cette absence de justification scientifique étayée poussa un certain nombre de chercheurs, et en particuliers les Russes, à proposer une autre limite basse, à savoir un hectare. La raison en est purement technique (détection ou déclaration administrative) : pour S. Rianjine, "puisque dans beaucoup de pays du monde (par exemple en Pologne, Bulgarie, Roumanie, au Japon, en Allemagne), les cadastres nationaux ne comptent pas les lacs d'une superficie inférieure à $0,01 \mathrm{~km}^{2}(1 \mathrm{ha})$, nous nous arrêterons à ce seuil de taille» (Rjanžin, 2005, en russe).

Le second écueil pour établir une méthodologie fiable est en lien avec les avancées

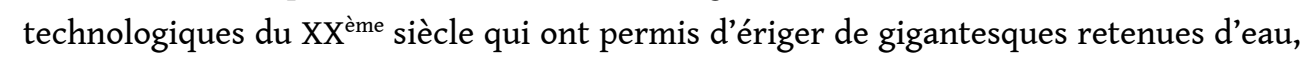
dont la masse liquide retenue en arrière de la digue est appelée «lac» en français ${ }^{2}$, mais également en lien avec l'abaissement ou non de la limite basse du lac. En ce cas, nombre de retenues nommées « étangs » en langue française entrent dans ce décompte. Si ces dernières n'influent en rien (ou presque) sur la limnicité mondiale, ce n'est pas le cas de retenues comme le lac des Trois Gorges en Chine, le lac de Bratsk en Russie ou encore le lac Mead aux Etats-Unis. 
15 Des choix sont donc à faire, mais ceux-ci doivent être motivés. Or, dans le cas des masses d'eau « plans d'eau » de la DCE, aucune logique précitée n'intervient :

- la limite basse des lacs naturels est fixée à 10 ha alors qu'au niveau mondial, le choix se limite à 1 ou 100 ha ;

17 - les « lacs » non naturels, appelés MEFM, sont pris en compte mais uniquement s'ils atteignent 50 ha (seuls ou à plusieurs). Là encore, la logique limnologique mondiale qui est de ne rien prendre en compte n'est pas respectée. Pour autant, cette décision dénote (au moins en nombre) l'importance prise par les retenues d'eau au sein de l'espace français.

18 Ainsi, si la limnicité offre un potentiel comparatif important sur la scène internationale, elle reflète mal les théâtres régionaux et surtout locaux où nombre de petites retenues d'eau parsèment les territoires. En fait, le concept a été créé par les Russes pour représenter uniquement la surface des plans d'eau naturels de plus d' 1 ha. Il pose donc la question de la place des plans d'eau artificiels. Bien que ces retenues que l'on nomme en français "étangs ", " mares ", " pêcheries ", ... soient en général d'une superficie infinitésimale par rapport aux lacs, leur multiplication voulue par les sociétés humaines doit interpeller quant à la pertinence de l'utilisation du terme «limnicité » comme marqueur de l'empreinte spatiale et sociétale des plans d'eau et non de la seule emprise spatiale.

19 Concernant la taille, les Russes possèdent depuis longtemps, à côté du terme d'oziornost, le terme de "zabolotchennost», qui se traduirait littéralement par «marécagicité », soit la part du territoire en marais, marécages, zones humides, en tout petits plans d'eau d'origine naturelle.

\section{De la « limnicité » à la « limnicité étendue » ou pourquoi étendre ce concept sémantique?}

20 La gestion de l'eau est au cœur des politiques économiques et environnementales dans de nombreux Etats à travers le monde. Cette gestion passe par une meilleure connaissance de la ressource en eau que ce soit quantitativement et qualitativement: une approche globale des plans d'eau est donc à privilégier, celle-ci sous-entendant la prise en compte des Hommes au sein de ces milieux.

21 Cette place anthropique de plus en plus prégnante demande donc d'élargir le champ des recherches à ce qui a été voulu par l'Homme et ne plus se limiter aux seuls objets naturels ${ }^{3}$.

\section{A. La limnicité est-elle représentative de la réalité lentique française?}

Comme indiqué précédemment, en 1995, M. Meybeck a avancé une limnicité stricto sensu de 0,09\% en France. Comparée aux 1,81 \% de moyenne mondiale, la limnicité française peut sembler faible. La raison est à chercher du côté des données climatiques passées et présentes, ainsi que de la tectonique. En effet, la plupart des étendues lacustres actuelles dans le monde sont le fait de barrages morainiques correspondant au front des grands inlandsis ou à des petites dépressions au sein de roches moutonnées. Le territoire métropolitain français n'ayant pas été sous la contrainte d'un 
inlandsis lors des dernières glaciations, seuls les massifs montagneux (Alpes, Jura, Vosges, Pyrénées, Massif Central et Corse) accueillent quelques lacs naturels dont le nombre et la superficie ne cessent naturellement de se réduire. La deuxième source de cuvette lacustre provient des mouvements de subsidence de l'écorce terrestre, soit sous forme de tectogenèse (rift rhénan) ou d'épeirogenèse entraînant la formation de dépressions pouvant donner lieu parfois à de l'endoréisme ; c'est le cas par exemple du lac de Grand-Lieu en Loire-Atlantique. Mais, dans le cas de la tectogenèse, celle-ci n'est plus suffisamment active actuellement pour permettre la constitution de dépressions lacustres comme a pu l'être la vallée de la Saône avec le fameux lac bressan. Ces fossés ont été comblés ce qui n'est pas le cas ailleurs avec par exemple le lac Baïkal ou le lac Tanganyika.

Cependant, si aux lacs naturels, nous ajoutons les lacs artificiels, la limnicité devient plus conséquente. En effet, l'inventaire général des plans d'eau français (Bartout \& Touchart, 2013) a permis de mettre en évidence une limnicité de 0,32 \%, chiffre conforme au résultat issu des travaux du GLWD effectués par photo-interprétation (Lehner \& Döll, 2004) avec 0,31 \%, certes toujours bien en deçà de la moyenne mondiale, mais suffisante pour que les plans d'eau n'apparaissent pas comme données négligeables dans l'occupation des sols de l'hexagone. La cartographie suivante montre également une certaine hétérogénéité des concentrations spatiales en France, car seules apparaissent distinctement le littoral languedocien et la Camargue, la côte landaise et les barrages en escaliers des vallées de la Dordogne et de la Truyère.

Figure 1 : La limnicité par commune en France en 2013.

Limnic ratio by commune in France in 2013.

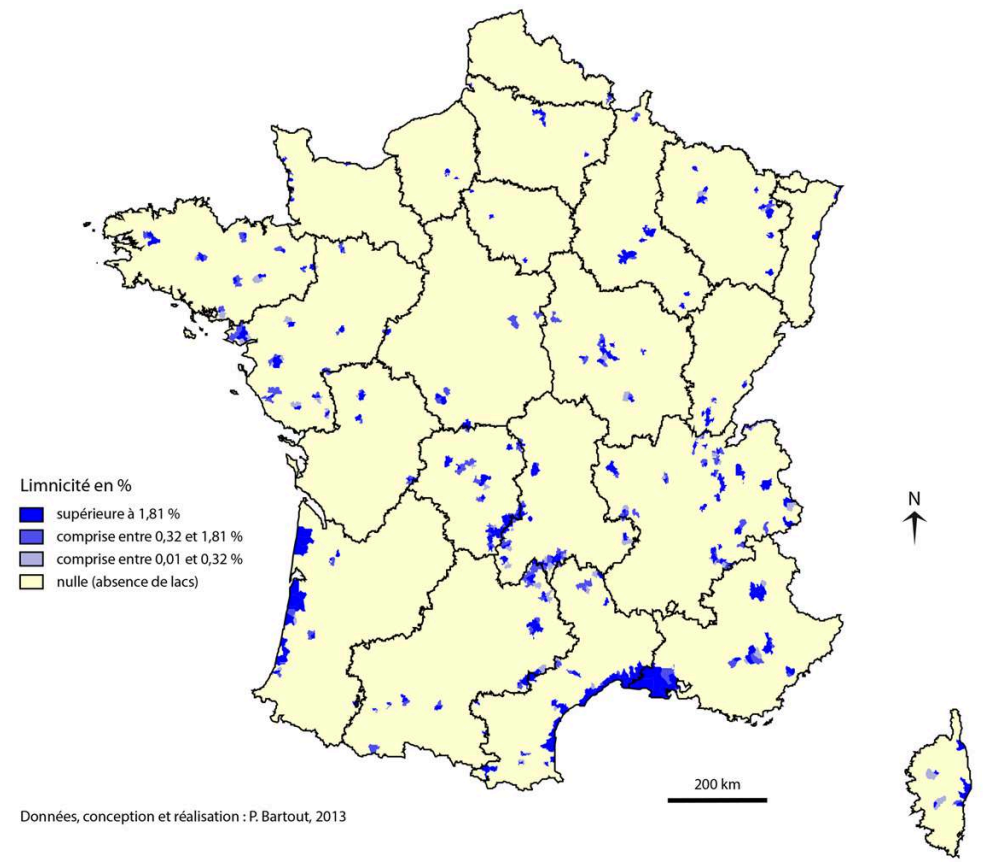

Mais cette recherche a montré également la faible place occupée surfaciquement par les lacs (un peu moins de $39 \%$ de la superficie lentique totale) dans l'Hexagone. Les lacs, qu'ils soient naturels, ou naturels et artificiels, sont donc minoritaires en étendue en France; une question se pose alors: doit-on prendre en considération les autres étendues lentiques? 


\section{B. La « limnicité étendue », un nouveau concept lentique.}

En prenant le parti de comprendre la catégorie surfacique première de chaque commune en France, nous avons produit la carte suivante qui met en évidence l'omnipotence des étangs sur plus des $3 / 4 \mathrm{du}$ territoire hexagonal. Omettre les étangs à l'échelle française reviendrait donc à ne pas comprendre la réalité lentique actuelle.

Figure 2 : La catégorie surfacique première des plans d'eau par commune en France en 2013. Water body's first surface area class by commune in France in 2013.

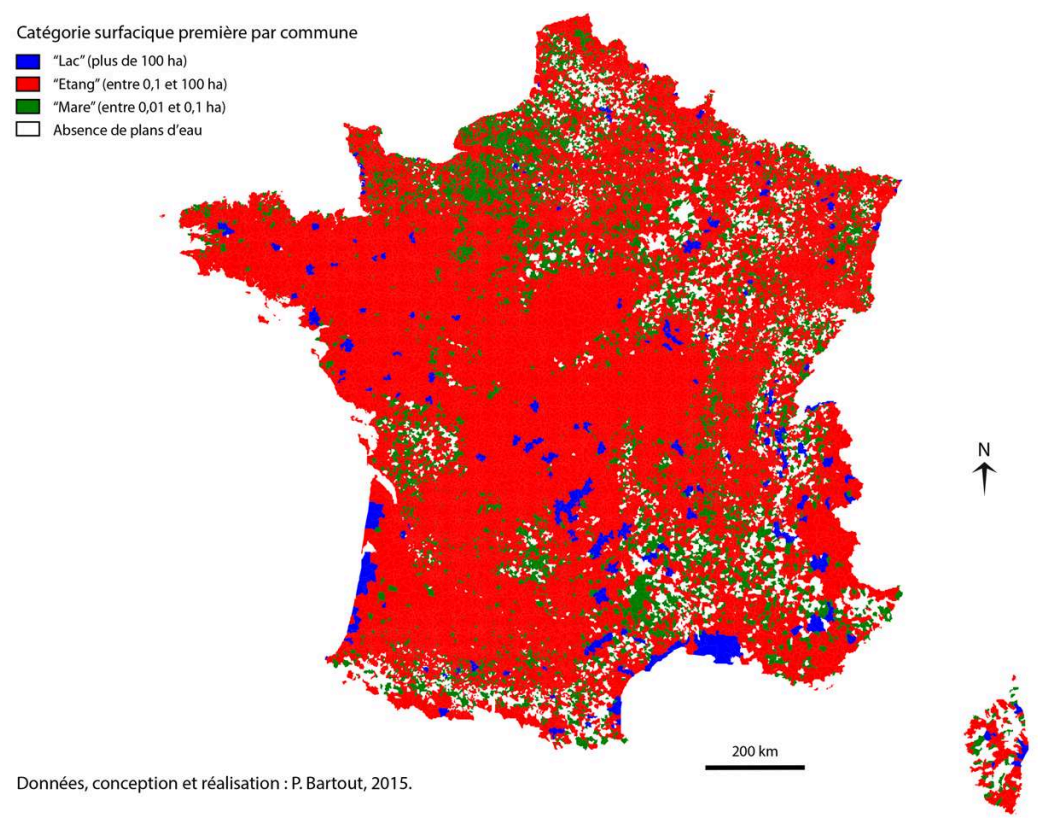

Cet aspect est très important pour l'approche scientifique et gestionnelle que les autorités en charge de la politique de l'eau hexagonale doivent avoir. En effet, se limiter aux seuls lacs procure certes des avancées indéniables et un rapport nombre d'études/limnicité sans équivalent avec les autres plans d'eau, mais ces travaux concernent une minorité de la composante limnique des milieux français. Anticipant cet état de fait dès 2010, nous avons créé le néologisme "stagnucité " permettant d'évaluer l'emprise spatiale des seuls « étangs " sur un territoire. D'abord appliquée à une région d'étangs, le Limousin $(2010,2012)$, cette approche a ensuite été généralisée à la France entière (Bartout \& Touchart, 2013). Même si le chiffre moyen de cette stagnucité peut sembler faible (autour de $0,47 \%$ en moyenne française), il peut dépasser localement les $20 \%$, notamment en Brenne ou en Dombes et surtout ce chiffre est 5 fois supérieur à l'estimation faite par M. Meybeck (1995) de la limnicité hexagonale.

Les travaux de l'américain J.A. Downing (Downing \& al, 2006, Downing \& Duarte, 2009, Downing, 2010), ont profondément modifié la perception du fait plan d'eau sur Terre. En effet, en 2006, sans proposition de changement conceptuel, c'est-à-dire en continuant de ne prendre en compte que les plans d'eau naturels, il a abaissé le seuil de détection à seulement 10 ares. Puis, en 2009, il introduit les plans d'eau artificiels dans son concept de limnicité, avant, en 2010, d'abaisser son seuil de détection à un are. 
- empreinte surfacique des lacs naturels (limnicité stricto sensu) : 0,09\% du territoire ;

- empreinte surfacique des lacs $^{5}$ (naturels comme artificiels) : 0,32 \% du territoire ;

- empreinte surfacique des étangs ${ }^{6}$ (naturels comme artificiels) : 0,47\% du territoire ;

- empreinte surfacique de tous les plans d'eau naturels et artificiels de plus d'un ha:

$0,68 \%$ du territoire ;

- empreinte surfacique de tous les plans d'eau naturels et artificiels de plus de dix ares :

$0,79 \%$ du territoire ;

- empreinte surfacique de tous les plans d'eau naturels et artificiels de plus d'un are (limnicité étendue) : 0,82\% du territoire.

Pour ce faire, ces chercheurs ont utilisé le «postulat de Wetzel » (1990) fondé sur une relation étroite entre la superficie et la quantité de lacs. Ils ont repris et perfectionné modele mathématique issu des travaux de M. Meybeck (1995a) en l'étendant aux inverse cette logique et considère que les très petits plans d'eau sont surfaciquement GLOWABO) ont rendu caduque cette estimation : pour Ch. Verpoorter et al (2014), les plans d'eau mondiaux de tous types et de plus de 0,2 ha (limite basse de la fiabilité des images Landsat utilisées) occuperaient 5 millions de $\mathrm{km}^{2}$, soit 3,7 \% des terres non englacées.

terme « limnicité ». Comme le demande L. Touchart (2014), « la notion de limnicité devient alors, peut-être, abusive et un autre terme conviendrait mieux». Nous proposons donc le avant précédemment : il s'agira alors de la part des plans d'eau à la surface, quelles que soient leurs dimensions ${ }^{4}$ et leurs origines.

Appliquée à la France, la limnicité étendue donne la cartographie suivante où les territoires mis en avant par la cartographie de la limnicité (figure 1) apparaissent totalement secondaires par rapport aux énormes concentrations de la Sologne, de la Vendée et du nord-ouest du Massif Central qui sont le fait avant tout d'étangs. 
Figure 3 : La limnicité étendue en France en 2013. Extended limnic ratio in France in 2013.

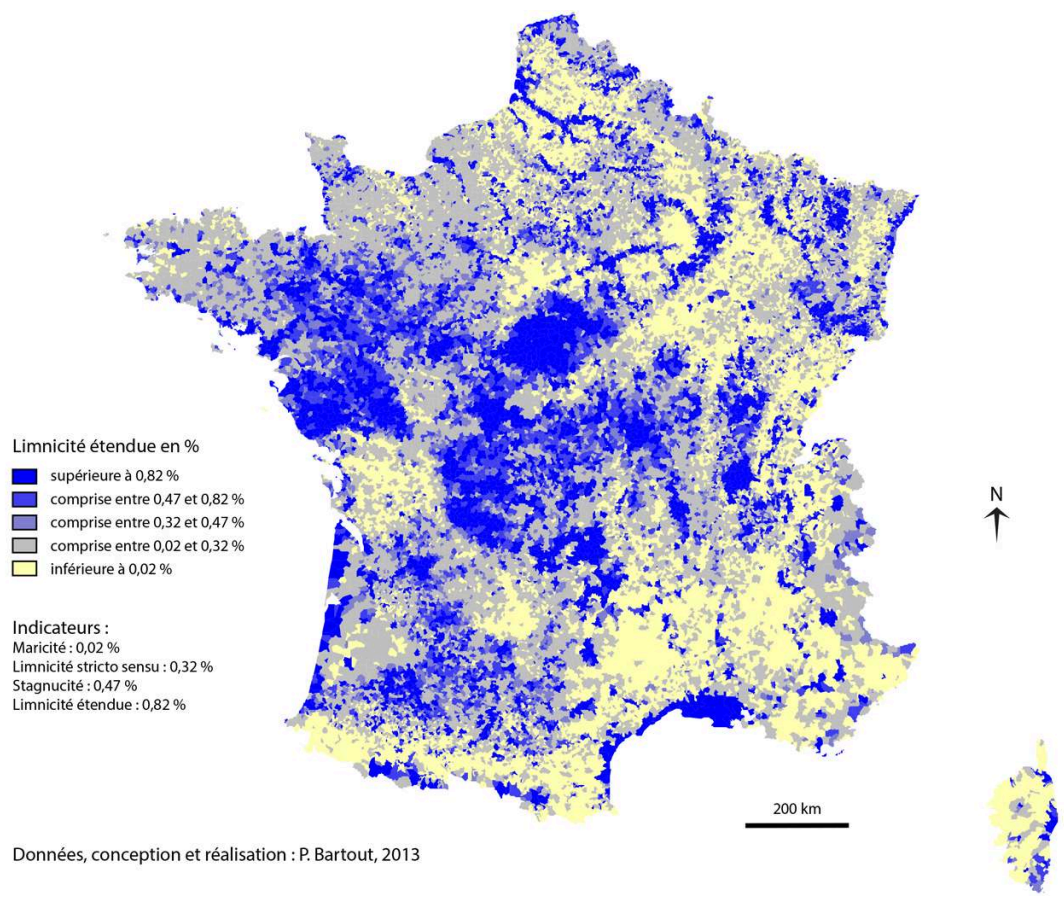

Si le géographe se positionne en tant que gestionnaire, la limnicité étendue répond davantage aux besoins actuels que la limnicité. Pour autant, la question de la prééminence de la superficie sur tous les autres facteurs afférents aux plans d'eau mérite d'être posée.

\section{La dimension spatiale est-elle encore le critère dominant de l'approche des plans d'eau?}

Les travaux touchant les définitions de plans d'eau (lacs, étangs, mares pour la langue française) ont démontré que restreindre la compréhension de phénomènes physicochimiques complexes au seul aspect surfacique étaient bien trop réducteurs. La plupart des limnologues, depuis F.-A. Forel (1895), accordent même davantage d'importance aux phénomènes verticaux à l'intérieur de la masse d'eau qu'à ceux horizontaux. En effet, ils mettent en évidence une structuration verticale de couches d'eau différenciées (nommées épilimnion, métalimnion et hypolimnion selon la terminologie classique de Birge, 1910) pouvant régulièrement être brassées en totalité selon un rythme saisonnier régulier dans les lacs. Dans le cas des étangs, moins profonds, L. Touchart (2002) qui a montré que la structuration verticale était dérangée selon un rythme irrégulier, au bout de quelques semaines ou quelques jours, a créé les néologismes d'épistagnion, métastagnion et hypostagnion (Touchart, 2007). Dans les mares, encore plus petites, le brassage diurne complet s'effectue à un rythme régulier. En conséquence, les césures sémantiques entre les trois termes définissant les masses d'eau stagnantes en langue française ont aujourd'hui tendance à s'orienter vers la notion de profondeur plutôt que vers celle de superficie, même si tous les auteurs s'accordent à dire que les deux ont leur importance. 

surfacique que sur une délimitation verticale car cette dernière nécessite une étude précise de la bathymétrie de chaque plan d'eau contrairement à la première où une simple image permet de renseigner grossièrement l'objet étudié. Mais cette simplification est-elle pertinente alors qu'il existe des impératifs de gestion?

\section{A. Plans d'eau et sociétés : une compréhension à réinventer.}

Aujourd'hui, en France, les plans d'eau sont avant tout petits et de création anthropique. Si le premier point a déjà été débattu dans cet article, le second entraîne des conséquences importantes sur le milieu. En effet, ces plans d'eau sont munis de dispositifs d'évacuation des eaux variés dont l'impact sur le milieu lotique aval est distinct (déversoir de surface, moine ou dérivation), aussi bien sur la température, l'oxygène dissous que les MES. Tant que ces étangs sont suffisamment éloignés les uns des autres, les caractéristiques de chaque système d'évacuation des eaux se cumulent après un retour à la normale par réajustement thermique et oxymétrique au sein du cours d'eau entre les deux objets. Mais, la trop grande proximité de ces plans d'eau modifie profondément la gestion de l'ensemble : ainsi l'impact thermique d'un étang de 10 hectares n'est pas le même que celui de 10 étangs de 1 hectare en chaîne. Lorsqu'ils sont munis d'un déversoir de surface, L. Touchart a quantifié l'impact du premier plan d'eau à $2^{\circ} \mathrm{C}$ annuels et $0,3^{\circ} \mathrm{C}$ supplémentaire pour chaque plan d'eau distant de moins de $300 \mathrm{~m}$. Le nombre de plans d'eau serait donc d'une importance considérable pour comprendre certains liens entre plans d'eau et cours d'eau qui sont au cœur des problématiques actuelles avec l'objectif $d u$ «bon état écologique ».

L'approche numérique internationale n'est pas absente du débat mais elle est beaucoup plus récente que l'approche surfacique. Ainsi, des chiffres existent à l'échelle du Monde (Meybeck, 1995, Downing \& al, 2006, Downing \& Duarte, 2009, Verpoorter \& al, 2014), de l'Europe (Kristensen \& Hansen, 1994) ou de la France, même s'ils sont en général très éloignés des réalités du terrain, surtout si l'on tient compte des plus petits d'entre eux qui sont sujets à tous types de croyances.

La particularité des modèles mathématiques en vogue en limnologie depuis un peu plus d'une décennie est de surestimer ces très petits plans d'eau. Cela influe fortement sur le chiffrage global : de 8,4 millions de « lacs » de plus d'un hectare en 1995 (Meybeck) dont 6,65 millions connus administrativement, nous sommes passés à 3,5 milliards de « plans d'eau » de plus d'un are en 2010 (Downing).

39 A l'opposé, les inventaires régionaux, nationaux et internationaux fondés sur des estimations de terrain sous-estiment en général ces très petits plans d'eau, y compris dans les grandes régions lacustres des hautes latitudes. Ainsi, en Estonie, il n'existerait officiellement qu'un peu plus de 800 plans d'eau de taille inférieure à 1 ha (Estonian Lakes Inventory, 2006).

40 En adoptant une démarche scientifique basée sur le local et progressivement élargie au global à partir du croisement de multiples méthodes, terrains et référentiels régionaux et nationaux (Bartout, sous presse), nous sommes parvenus à fiabiliser davantage ces bases de données.

41 Ainsi, en Estonie, par l'intermédiaire du programme franco-estonien PARROT (2013-2014) entre les universités d'Orléans et de Tallinn, nous avons mis en évidence plus de 86500 plans d'eau (Bartout \& al, sous presse). A l'échelle de l'Europe, nous

Revue Géographique de l'Est, vol.56 / n 1-2 | 2016 
évaluons le nombre de plans d'eau à 4,45 millions (dont 591000 de plus d'un hectare), au lieu des 500000 plans d'eau qui seraient présents d'après les chiffres produits par l'EEA (Agence Européenne de l'Environnement) et repris par les travaux de P. Kristensen et H.O. Hansen (1994). A l'échelle mondiale, nos estimations varient entre 200 et 400 millions d'objets lentiques de plus de $100 \mathrm{~m}^{2}$ (Terasmaa \& al, 2015, Bartout \& al, sous presse) dont 117 millions sont parfaitement localisés (Verpoorter \& al, 2014).

Toutefois, la dominante des plans d'eau de superficie inférieure à un hectare est un fait mondial ( $76,5 \%$ des plans d'eau mondiaux, 86,7 \% des plans d'eau européens et 91,7 \% des plans d'eau français) qui nécessite un ajustement des choix des gestionnaires de l'eau. Même au-delà de ce seuil d'un hectare, les panels lentiques diffèrent comme les tableaux 1 et 2 ci-après le montrent. Nous y avons confronté différents inventaires limniques aux trois échelles développées jusqu'à présent : France, Europe et Monde.

Tableau 1 : Le nombre de lacs (en milliers) en France, en Europe et dans le monde. Lake's number in France, Europe and the world.

\begin{tabular}{|l|l|l|l|l|l|l|l|l|l|l|l|}
\hline \multirow{2}{*}{ Auteur } & Publié en & \multicolumn{2}{l|}{ Nombre France } & \multicolumn{2}{l|}{ Nombre Europe } & \multicolumn{2}{l|}{ Nombre Monde } \\
\cline { 3 - 12 } & & & +1 ha & +10 ha & +100 ha & +1 ha & +10 ha & +100 ha & +1 ha & +10 ha & +100 ha \\
\hline $\begin{array}{l}\text { Kristensen \& } \\
\text { Hansen }\end{array}$ & 1994 & & & & 500 & & 16 & & & \\
\hline Meybeck & 1995 & & & & & & & 8440 & 1240 & 14 \\
\hline Downing \& al & 2006 & & & & & & & 26416 & 2296 & 200 \\
\hline Bartout & 2013 & 46,1 & 3,7 & 0,3 & & & & & & & \\
\hline Verpoorter \& al & 2014 & & & & & & & & 27523 & 4123 & 353 \\
\hline Bartout & 2015 & & & & 591 & 125 & 18 & & & \\
\hline
\end{tabular}

Tableau 2 : Comparaison des classes surfaciques de lacs entre des approches française, européenne et mondiale.

Comparison of lake's surface area between French, European and world approaches.

\begin{tabular}{|c|c|c|c|c|c|}
\hline & $\begin{array}{l}\text { Nombre de } \\
\text { «lacs 》 de plus } \\
\text { de } 1 \text { ha }\end{array}$ & $\begin{array}{c}\text { Nombre de } \\
\text { "lacs 》 de plus de } \\
10 \text { ha }\end{array}$ & $\begin{array}{c}\text { Part des « lacs» de plus } \\
\text { de } 10 \text { ha à l'intérieur de } \\
\text { l'ensemble de plus de } 1 \\
\text { ha }\end{array}$ & $\begin{array}{l}\text { Nombre de } \\
\text { «lacs » de plus de } \\
100 \text { ha }\end{array}$ & $\begin{array}{l}\text { Part des « lacs » de } \\
\text { plus de } 100 \text { ha à } \\
\text { l'intérieur de } \\
\text { l'ensemble de plus de } \\
\text { l ha }\end{array}$ \\
\hline $\begin{array}{c}\text { Monde } \\
\text { (Verpoorter \& al, } \\
\text { 1995) }\end{array}$ & 27523000 & 4123000 & $14,98 \%$ & 353000 & $0,13 \%$ \\
\hline $\begin{array}{c}\text { Europe } \\
\text { (Bartout, 2015) }\end{array}$ & 591394 & 125479 & $21,22 \%$ & 17615 & $0,30 \%$ \\
\hline $\begin{array}{c}\text { France } \\
\text { (Bartout, 2013) }\end{array}$ & 46140 & 3677 & $7,97 \%$ & 292 & $0,63 \%$ \\
\hline
\end{tabular}

Ces résultats mettent en avant une spécificité du corpus lentique français que la limnicité seule ne pouvait montrer. En effet, la France souffre d'une sousreprésentation nette des plans d'eau de superficie comprise entre 10 et 100 ha alors qu'elle possède une surreprésentation pour ceux de superficie comprise entre 1 et 10 ha. Ces faits tiennent autant à la faiblesse quantitative des lacs naturels qu'à celle de la valorisation économique des «étangs» français, principalement tournés vers l'agrément individuel et non vers une rentabilité économique telle que la pisciculture. La surreprésentation des plans d'eau de plus de 100 ha est en revanche typique d'états où l'édification de lacs de barrage a constitué au cours du $20^{\text {ème }}$ siècle un moyen de réguler les régimes des cours d'eau.

Cette relative faiblesse concernant les plans d'eau moyens en France se vérifie également au niveau de la superficie puisque cette classe se positionne derrière les plus de 100 ha (47,17 \%) mais également derrière ceux dont la superficie est comprise entre 1 et 10 ha (respectivement $29,76 \%$ contre $23,07 \%$ ).

Afin de comparer spatialement les approches numériques et surfaciques, la figure 4 a été construite en se basant sur la méthode du quartile. Elle représente les densités de 
plans d'eau de plus d'un hectare par commune française, permettant d'identifier les secteurs où ce type de retenues est fréquent, voire très fréquent, puisque l'on dépasse par endroit la densité de 5 plans d'eau par $\mathrm{km}^{2}$. Ces secteurs sont pour certains différents de ceux identifiés précédemment (figure 3) puisque apparaissent très nettement les fortes concentrations de Dombes, Brenne, Sologne, Sologne bourbonnaise, Camargue ou Cantal, alors que la Vendée et nord-ouest du Massif Central sont moins prééminents compte tenu de la petitesse de leurs plans d'eau.

Figure 4 : La densité de plans d'eau de plus de un hectare par commune en France. The density of water bodies more than one hectare by commune in France.

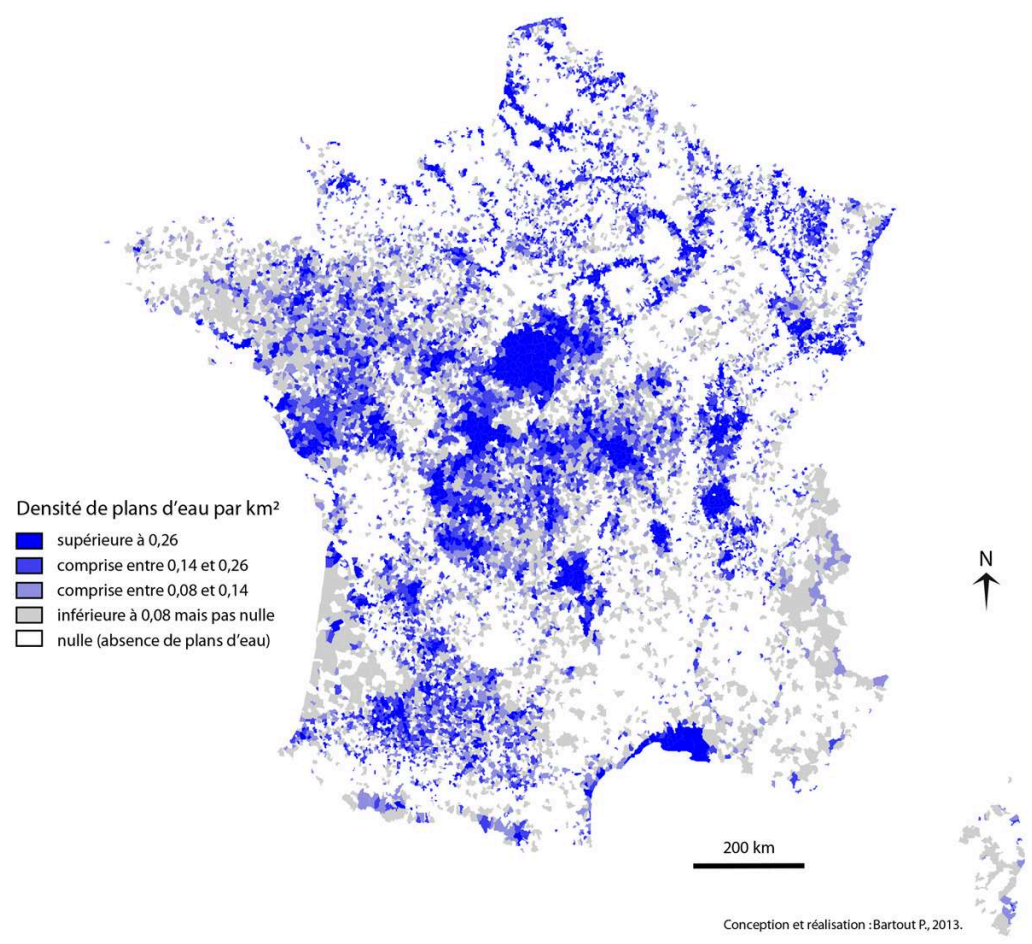

Cette petitesse est encore plus mise en exergue par cette carte des densités de plans d'eau, tous types confondus, intégrant de fait les petits étangs et l'ensemble des mares de plus de $100 \mathrm{~m}^{2}$. La régionalisation lentique diffère des enseignements tirés de la figure 4 avec la mise en avant de territoires méconnus en limnologie qui ont la particularité de se trouver tous dans le Grand Ouest français, dont le point commun est avant tout d'abriter des structures agraires bocagères. 
Figure 5 : La densité de plans d'eau par commune en France, tous types confondus. The density of all water bodies by commune in France.

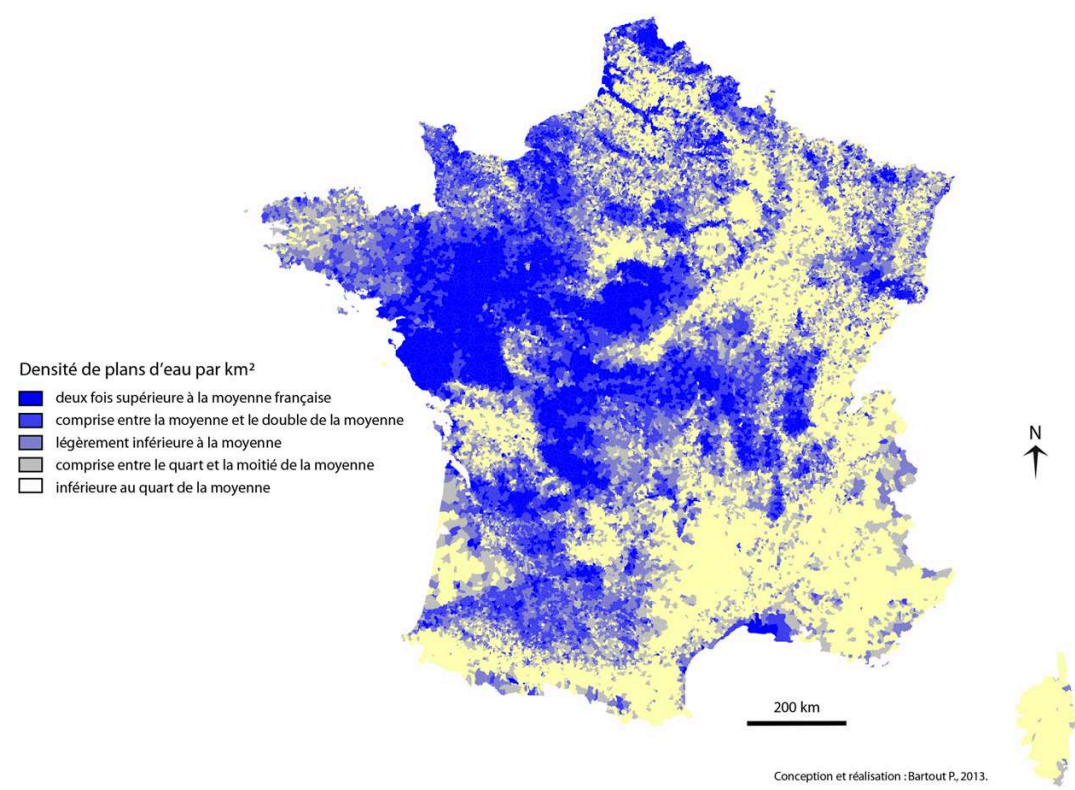
chaque plan d'eau. Elle met également en évidence des différenciations régionales fortes entrainnant par là même des besoins et des modes de gestion différenciés. Cette approche sociale et sociétale mérite donc d'être approfondie.

\section{B. La mise en place de nouveaux indicateurs lentiques pour mieux appréhender leur rôle dans le milieu.}

A l'échelle départementale, qui est l'échelle de gestion actuelle des plans d'eau par l'intermédiaire des Directions Départementales des Territoires (DDT), nous avons essayé d'imaginer un indicateur qui permettrait de faire apparaître la complexité de la gestion des plans d'eau pour une entité administrative. Compte tenu du peu de moyens financiers et humains à disposition, nous sommes partis du principe qu'une gestion lentique ne pouvait être efficace que si le fait « plan d'eau » était extrêmement localisé. De fait, pour quantifier cette dispersion spatiale, nous avons donné naissance à deux indicateurs qui permettent à la fois de savoir si le fait «plan d'eau » était général dans un département ou simplement localisé (ce qui en facilite la gestion) et si une région bien précise du département écrasait les autres, ce qui facilitait également la gestion humaine en concentrant les moyens sur cette zone : 
décrochage statistique, celui-ci apparaissait dans la plupart des cas entre le $3^{\text {ème }}$ et le 8 ème centile.

52 Cet IEC se lit donc de la manière suivante : sa valeur maximale est 0,95 (dans le cas où toutes les communes d'un département possèderaient des plans d'eau ${ }^{7}$ et où chacune d'entre elles possèderait un nombre équivalent de plans d'eau) et sa valeur minimale est fixée à $-0,99$ (dans le cas où il n'y ait qu'une seule commune à accueillir un ou des plans d'eau). Plus l'indicateur est élevé et plus leur gestion à l'échelle départementale est complexe.

L'IES est construit sur le même principe sauf qu'il s'agit de remplacer le nombre de plans d'eau par la superficie qu'ils occupent.

Figure 6 : L'indicateur d'égale concentration des plans d'eau en France. Water body's equal concentration subscript (ECS) in France.

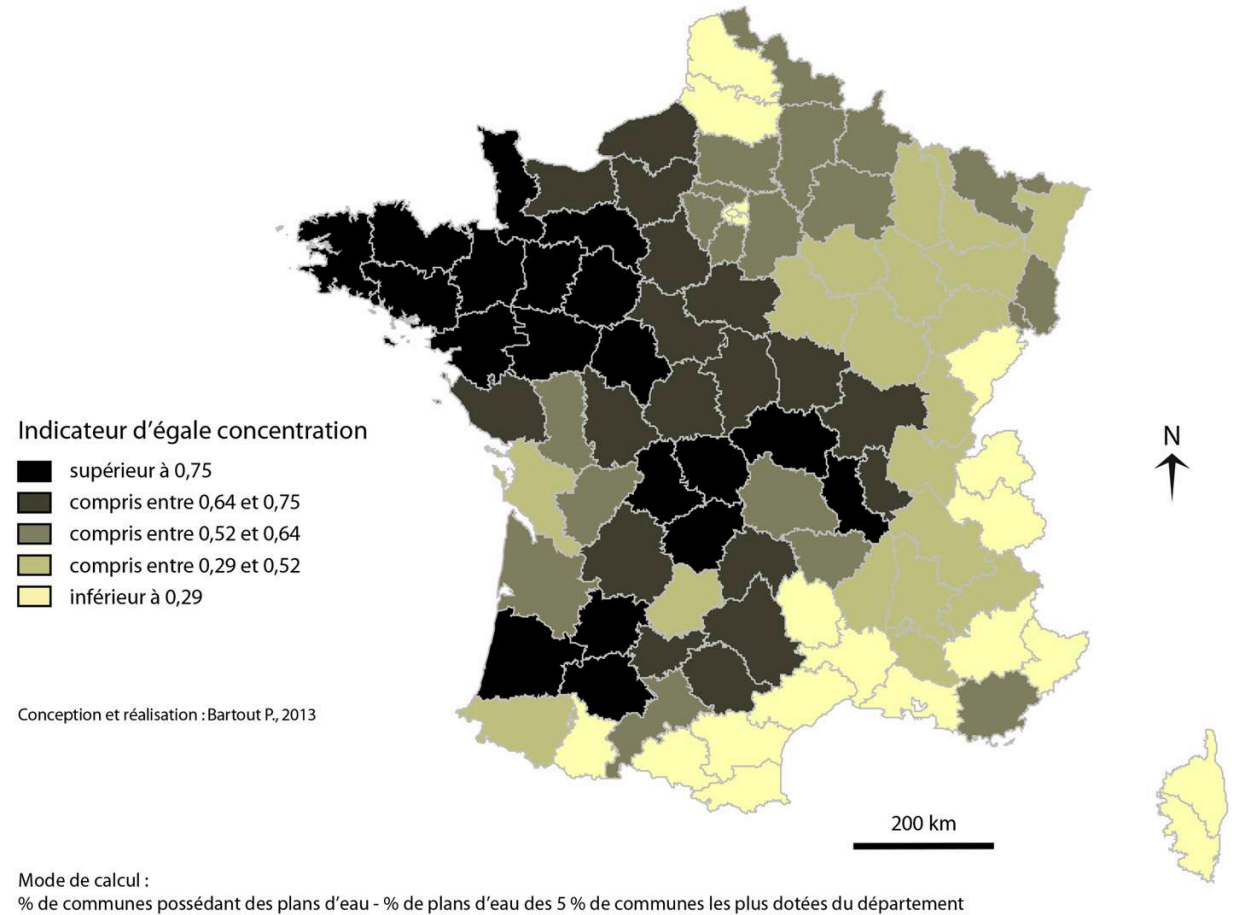


Figure 7 : L'indicateur d'égale superficie des plans d'eau en France. Water body's equal surface area subscript (ESS) in France.

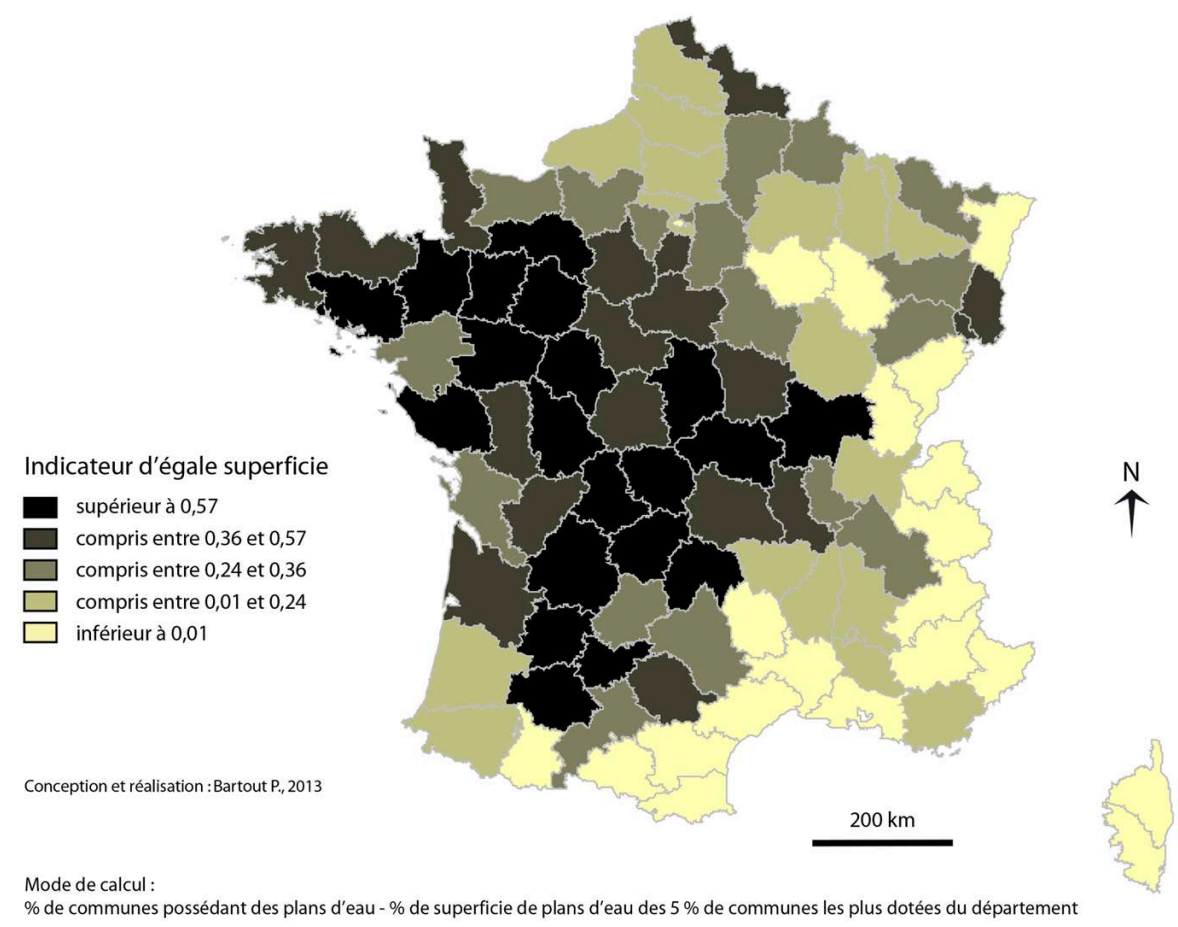

$$
\text { Mass }
$$

\section{Mas} - pour l'IEC, différentes auréoles apparaissent : un très fort indice pour l'Ouest (excepté
le golfe de Charente) et le Centre-Ouest avec trois pôles (Massif armoricain, Nord du Savoie, mais également vers la Picardie et l'Artois au Nord, et des indices très faibles dans les massifs calcaires et le milieu méditerranéen.

- Pour l'IES, même si la domination de l'Ouest reste visible, le dégradé W/E apparaît moins linéaire, les plus forts indices reculant légèrement à l'intérieur des terres, alors que la Champagne remplace la Picardie pour ces faibles indices. En revanche, les massifs calcaires et le milieu méditerranéen conservent un très faible indice. 
Tableau 3 : Classement des 10 plus forts et 10 plus faibles IEC et IES en France. The ten firsts and ultimate ECS and ESS rankings in France.

\begin{tabular}{|c|c|c|c|c|c|}
\hline \multicolumn{3}{|c|}{ Indicateur d'Egale Concentration } & \multicolumn{3}{|c|}{ Indicateur d'Egale Superficie } \\
\hline Classement & Département & Indice & Classement & Département & Indice \\
\hline 1. & Mayenne & 0,867 & 1. & Mayenne & 0,789 \\
\hline 2. & Allier & 0,834 & 2. & Lot-et-Garonne & 0,781 \\
\hline 3. & Creuse & 0,831 & 3. & Allier & 0,761 \\
\hline 4. & Morbihan & 0,829 & 4. & Haute-Vienne & 0,750 \\
\hline 5. & Maine-et-Loire & 0,828 & 5. & Maine-et-Loire & 0,738 \\
\hline 6. & Haute-Vienne & 0,826 & 6. & Tarn-et-Garonne & 0,725 \\
\hline 7. & Sarthe & 0,824 & 7. & Indre-et-Loire & 0,722 \\
\hline 8. & Lot-et-Garonne & 0,819 & 8. & Sarthe & 0,679 \\
\hline 9. & Orne & 0,808 & 9. & Ille-et-Vilaine & 0,671 \\
\hline 10. & Ille-et-Vilaine & 0,803 & 10. & Vendée & 0,669 \\
\hline 87. & Pyrénées Orientales & 0,036 & 87. & Ariège & $-0,192$ \\
\hline 88. & Ariège & 0,027 & 88. & Doubs & $-0,196$ \\
\hline 89. & Hauts-de-Seine & 0,015 & 89. & Lozère & $-0,213$ \\
\hline 90. & Hautes-Pyrénées & $-0,048$ & 90. & Hautes-Pyrénées & $-0,216$ \\
\hline 91. & Alpes-Maritimes & $-0,092$ & 91. & Seine & $-0,223$ \\
\hline 92. & Gard & $-0,119$ & 92. & Alpes-Maritimes & $-0,322$ \\
\hline 93. & Corse-du-Sud & $-0,120$ & 93. & Pyrénées-Orientales & $-0,341$ \\
\hline 94. & Val-de-Marne & $-0,192$ & 94. & Corse-du-Sud & $-0,346$ \\
\hline 95. & Seine & $-0,268$ & 95. & Gard & $-0,386$ \\
\hline 96. & Haute-Corse & $-0,281$ & 96. & Haute-Corse & $-0,611$ \\
\hline
\end{tabular}

Ce tableau 3 vient encore plus renforcer l'idée d'une régionalisation marquée du fait lentique en France, puisque 7 des 10 premiers du classement de l'IEC (en italique dans le tableau) sont également présents aux premières places de l'IES et 8 des 10 derniers au classement de l'IEC se retrouvent aux mêmes places en IES.

Le Massif Armoricain et le Limousin apparaissent comme les secteurs où la gestion des plans d'eau s'avère être la plus complexe car ils sont présents partout et sans réelles concentrations écrasant les autres. A l'opposé la bordure méditerranéenne, les Pyrénées centrales, les Alpes et le Jura sont des territoires où l'on assiste à une ultra concentration $d u$ fait lentique rendant plus simple une gestion adaptée puisque beaucoup plus localisée.

Le dernier enseignement de ces figures est la place secondaire occupée par les départements possédant les régions de plans d'eau les plus reconnues nationalement et internationalement et sur lesquels les modes de gestion actuels sont calés : moyen pour l'Indre et le Loir-et-Cher (Brenne et Sologne), faible pour l'Ain, la Meurthe-et-Moselle, la Moselle et la Meuse (Dombes, Woëvre, Argonne...), voire très faible pour les plans d'eau littorau avec les Bouches-du-Rhône, le Gard, l'Hérault et les Pyrénées Orientales (Camargue, Languedoc). Ce constat montre combien il existe de décalages entre la réalité de terrain des plans d'eau actuellement en France et ce qu'on voudrait qu'elle fût.

\section{Conclusion}

60 La notion de limnicité, reflet d'une approche partielle des milieux lentiques dans le monde, apparait aujourd'hui comme désuète par rapport aux enjeux dans le domaine de l'eau. Ceux-ci touchent règlementairement au respect des critères du «bon état écologique » de l'eau énoncés dans la DCE-2000.Or ces critères d'ordres physicochimiques, morphologiques et sédimentaires visent à limiter l'influence des activités humaines sur le milieu sans tenir compte de ce que, dans la plupart des cas, c'est la 
société elle-même du pays très anciennement humanisé qu'est la France qui est à l'origine de l'écosystème actuel.

A partir du moment où l'on décide d'entrer dans une politique de gestion d'un milieu et non de sa seule compréhension, le terme et ses actions inhérentes doivent évoluer pour tenir compte d'une plus grande variété et disparité spatiale, mais aussi économique, fonctionnelle, écologique (Bouleau \& Pont, 2014). La place dominante en France des plans d'eau culturels sur ceux naturels, qui sont ceux d'où sont tirées les normes limnologiques, oblige à repenser le choix des indicateurs et à les adapter à cette pluralité lentique.

géographe se positionne en tant que gestionnaire, la limnicité étendue répond davantage aux besoins actuels que la limnicité. Ainsi, au niveau des deux Etats présentés dans cet article, à savoir l'Estonie et la France, et au regard de la quantité de plans d'eau en fonction des critères de "limnicité » et "limnicité étendue ", nous obtenons des valeurs très différentes.

De la sorte, dans un territoire où la présence de grands lacs est attestée comme l'Estonie, la prise en compte des 86500 plans d'eau au lieu des 2800 lacs précédents (Estonian Lakes Inventory, 2006) ne fait progresser que de 7,5\% la valeur de la «limnicité étendue » par rapport à la « limnicité » précédente (de 4,82 \% à 5,18 \%) malgré 30 fois plus de plans d'eau pris en compte. A l'inverse, sur un territoire dénué de grands lacs comme la France, la prise en compte de tous les plans d'eau modifie beaucoup plus largement la perception surfacique du «fait limnique»: d'une «limnicité » de 0,09\% avec 300 lacs comptabilisés, nous passons à une «limnicité étendue » de $0,82 \%$ avec plus de 554000 plans d'eau, soit $900 \%$ d'augmentation avec 1900 fois plus de plans d'eau. Cette dichotomie entre l'Estonie $(+7,5 \%$ avec une hausse de la valeur brute de la « limnicité » de 0,36\%) et la France (+ $900 \%$ avec une hausse de $0,73 \%$ soit deux fois celle estonienne) nous montre qu'il existe une réelle différence de connaissances du corpus limnique entre ces deux Etats et que la compréhension des seuls grands plans d'eau phagocyte donc les perceptions limnologiques en empêchant de comprendre les particularismes locaux des plans d'eau.

Cette approche par la superficie en eau, si elle reste représentative d'une certaine réalité lentique, doit donc s'accompagner de la mise en place d'autres indicateurs pertinents, dont l'objectif est de comprendre la place de l'Homme dans ces milieux permettant un équilibre Homme/Nature si recherché.

De fait, aujourd'hui, cet équilibre n'est pas pris en compte pour les plans d'eau artificiels, notamment les plus petits comme les étangs. Pour autant, B. Clément et A. Aidoud (2015)écrivent que « les étangs accueillent $30 \%$ des espèces protégées à statut national ou régional alors que les zones humides en général ne représentent que $3 \%$ du territoire national: les étangs ont donc une valeur patrimoniale indubitable avec un rapport de $1 \grave{a} 10$ ». La création anthropique est certes source de modifications du milieu, mais elle crée également un potentiel écologique avec l'apparition de nouveaux biotopes susceptibles d'augmenter la biodiversité. Nier ce fait revient à contester le patrimoine matériel et immatériel associé à ces milieux humides, leur histoire, leur économie..., bref leurs rôles social et sociétal.

Enfin, cette nouvelle acception doit permettre de réenvisager la place des plans d'eau autres que les lacs naturels dans la recherche française, mais plus encore dans la recherche en général, en discernant notamment des périmètres de gestion divers tels 
que les bassins-versants, les hydro-écorégions (Wasson \& al, 2004) ou les limnorégions (Bartout, 2015).

\section{BIBLIOGRAPHY}

Bartout P., 2010, Pour un référentiel des zones humides intérieures de milieu tempéré - l'exemple des étangs en Limousin (France), Sarrebruck, Editions Universitaires Européennes, 456 p.

Bartout P., 2012, Les étangs du Limousin : des zones humides face au développement durable, Brive-laGaillarde, Les Monédières, 250 p.

Bartout P., 2015, Les territoires limniques. Nouveau concept limnologique pour une gestion géographique des milieux lentiques, Mémoire HDR de géographie, Université d'Orléans, 444 p.

Bartout P., sous presse, «L'incompréhension de la place prise par les plans d'eau dans l'Union Européenne et ses conséquences règlementaires ", Norois, 235, 35 p.

Bartout P., Touchart L., 2013, «L'inventaire des plans d'eau français : outil d'une meilleure gestion des eaux de surface », Annales de Géographie, 691, p. 266-289.

Bartout P., Touchart L., Choffel Q., Terasmaa J., Koff T., Kapanen G., Qsair Z., Marzecova A., Saudubray J., Aldomany M., Millot C., Maleval V., sous presse, “A new approach to inventories of bodies of water, from local to global scale", Die Erde, 16 p.

Birge E.A., 1910, « A unregarded factor in lake temperatures ", Transactions of the Wisconsin Academy of Sciences, Arts and Letters, 16, p. 989-1004.

Bouleau G., Pont D., 2014, «Les conditions de référence de la directive cadre européenne sur l'eau face à la dynamique des hydrosystèmes et des usages ", Natures Sciences Sociétés, 22, p. 3-14.

Brochu M., Michel J.-P., 1994, Dictionnaire de géomorphologie à caractère dimensionnel, Paris, Guérin, 298 p.

clément B., Aidoud A., 2015, « Oligotrophie du substrat et marnage, une combinaison de caractéristiques propre à contrôler l'invasibilité des systèmes palustres ", in Touchart L., Bartout P., Motchalova O. (dir), Mieux comprendre les étangs : expériences nationales et internationales, du Berry limousin à l'Europe orientale, Brive-la-Gaillarde, Les Monédières, p. 233-244.

Delebecque A., 1898, Les lacs français, Paris, Chamerot \& Renouard, 436 p.

Downing J.A., 2010, "Emerging global role of small lakes and ponds: little things mean a lot", Limnetica, $29, \mathrm{n}^{\circ} 1$, p. 9-24.

Downing J.A., Duarte C.M., 2009, « Abundance and size distribution of lakes, ponds and impoundments », in Likens G.F. (dir), Encyclopedia of inland waters, Amsterdam, Elsevier, p. 469-478.

Downing J.A., Cole J.J., Middelburg J.J., Striegl R.G., Duarte C.M., Kortelainen P., Prairie Y.T., Laube K.A., 2008, "Sediment organic carbon burial in agriculturally eutrophic impoundments over the last century”, Global Biogeochemical Cycles, 22, p. 1018. 
Downing J.A., Prairie Y.T., Cole J.J., Duarte C.M., Tranvik J.J., Striegl R.G., McDowell W.H., Kortelainen P., Caraco N.F., Melack J.M., Middelburg J.J., 2006, “The global abundance and size distribution of lakes, ponds and impoundments”, Limnology and Oceanography, 51, n5, p. 2388-2397.

Estonian Lakes Inventory, 2006, Eesti järvede nimestik. - Looduslikud ja tehisjärved, ed Ruta Tamre, Keskkonnaministeeriumi Info - ja Tehnokeskus.

Forel F.-A., 1895, Le Léman, monographie limnologique, Lausanne, F. Rouge, tome II, 651 p.

Halbfass W., 1933, « Seen » in Hess H. (dir), Das Eis der Erde, Berlin, Bornträger, Handbuch der Geophysik, Band 7, p. 122-182.

Halbfass W., 1934, « Der Jahreswasserhauhalt der Erde. Ist er quantitative eine konstante Grösse ?», Pettermanns Mitteilungen, 80, p. 137-140 \& 177-179.

Herdendorf C.E., 1982, « Large lakes of the world », Journal of Great Lake Research, 8, p. 379-412.

Herdendorf C.E., 1990, « Distribution of the world's large lakes », in Tilzer M.M., Serruya C. (dir), Large lakes, ecological structure and function, Berlin, Springer, p. 3-38.

Kristensen P., Hansen H.O. (dir), 1994, European rivers and lakes, assessment of their environmental state, Copenhaguen, European Environment Agency, 122 p.

Lehner B., Döll P., 2004, "Development and validation of a global database of lakes, reservoirs and wetlands". Journal of Hydrology, 296, p. 1-22.

Meybeck M., 1995a, « Les lacs et leur bassin », in Pourriot R., Meybeck M. (dir), Limnologie générale, Paris, Masson, p. 6-59.

Meybeck M., 1995b, « Global distribution of lakes », in Lerman A., Imboden D., Gat J. (dir), Physics and chemistry of lakes, Berlin, Springer, 2nd Ed, p. 1-35.

Penck A., 1894, « Die Wannen » \& « Die Wannen und Seenländer », in Morphologie der Erdoberfläche, Stuttgart, J. Engelhorn, zweiter Teil, p. 203-232 \& 233-327.

Rjanžin S.I., 2005, « Mnogo li na Zemle ozër? », Priroda, 4, p. 18-25.

Tamrazyan G.P., 1974, « Total lake water resources of the planet », Bulletin of the Geological Society of Finland, 46, p. 23-27.

Terasmaa J., Bartout P., Marzecova A., Touchart L., Koff T., Choffel Q., Kapanen G., Maleval V., Millot C., Qsair Z., Vandel E., 2015, "European perspectives on regional estimates of standing water bodies and the relevance of man-made ponds", Vienne, EGU Conference, poster.

Touchart L., 2002, Limnologie physique et dynamique, une géographie des lacs et des étangs, Paris, L'Harmattan, 395 p.

Touchart L., 2007, «La définition de l'étang en géographie limnologique » in Géographie de l'étang, des théories globales aux pratiques locales, Paris, L'Harmattan, p. 13-53.

Touchart L., 2014, Les territoires de l'eau en Russie, Paris, L'Harmattan, 331 p.

Trëšnikov A.F., 1988, Geografičeskij ènciklopedičeskij slovar', Moskva, Sovetskaja ènciklopedija, $432 \mathrm{p}$.

Verpoorter C., Kutser T., Seekell D.A., Tranvik L.J., 2014, “A global inventory of lakes based on high-resolution satellite imagery". Geophysical Research Letters, 41. 
Wasson J.G., Chandesris A., Pella H., Blanc L., 2004, « Les hydro-écorégions : une approche fonctionnelle de la typologie des rivières pour la Directive cadre européenne sur l'eau », Ingénieries-Eau Agriculture Territoires, 40, p. 3-12.

Wetzel R.G., 1990, “Land-water interfaces: Metabolic and limnological regulators". Int. Verein. Theor. Limnol. Verh, 24, p. 6-24.

Wetzel R.G., 2001, Limnology, lake and river ecosystems, San Diego, Academic Press, 3rd Ed, 1006 p.

\section{NOTES}

1. Ozërnost' en transcription internationale.

2. En revanche, les Anglo-Saxons répugnent à employer le mot «lake» dans ce cas, pour lui préférer presque toujours le terme de «reservoir». De même, en russe, «ozero » n'est que le lac naturel ; le lac de barrage est « vodohranilišče ».

3. Cette notion de naturalité étant d'ailleurs assez subjective : nous entendrons ici par «naturel » la création qui n'est pas du fait de l'Homme.

4. Dans les faits, une limite basse existe, à savoir 1 are, compte tenu de l'incertitude d'existence des plans d'eau de superficie inférieure.

5. Plus de 100 ha.

6. Entre 0,1 et 100 ha.

7. Ce qui est le cas de trois d'entre eux : le Maine-et-Loire, la Sarthe et la Haute-Vienne.

\section{ABSTRACTS}

The present article has for aim to discuss genesis and limits of « limnicity » notion in relation to new social and societal problems, arise from many lacks of understanding between legislator and ground actors about water masses notions of ECD law.

Those limits are numerous and already visible even in the structure of actually water's layout tools. Indeed, as part of ECD law application, French water agencies take into account "MEFM » (water masses which are so much modified) besides lakes even though they aren't naturals. So, it's a first transgression. It added to this the area threshold: without any limnic argument, this one is fixed at 50 hectares, while bidder the liberty of interpretation considering local realities (reduction of threshold, lacustrian regions).

Those choices have two consequences: firstly, the criterion of single lacustrian naturalist is jeopardize and the area arbitrary leads to a conflict on ground between water actors (less than 1 $\%$ of French water bodies are identified, the rest being considered by default as river section); secondly, the priority criterion of area is called into question by numeric notion specific to lacustrian regions.

The authors intend to adapt the "limnicity" notion to the lentic complexity and take into account all the lakes and man-made seas and, in parallel, increase the range of water bodies studied in integrating ponds which, at the scale of France, have a higher total area than that of lakes: so a new notion arises of this, the « extended limnic ratio ». 
Then, the choice of water body's spatial scope for dominant criterion is debated over linked with scientists progressives which highlight the pregnancy of lacustrian fact rehearsal compared to the impact of only one limnic object, whether it is the physical chemistry factors of water or societal plan. Also, water body's density is placed on the same analysis level than the water body's surface area. Thanks to this scientist development, two new indicators are produced allowing bettering understanding the role of water bodies in the environment: the equal surface area subscript (ESS) and the equal concentration subscript (ECS). Applied to French department scale, those two indicators highlight a major fact: the department which own the more recognizes lacustrian regions as well national as international, and which ones actually managements are decided, are secondary for the comprehension of social and environmental links existing actually at the water body's level. This analysis shows how much there is discrepancies between water body's ground reality and those imagined, and the only one notion of « limnicity » can't be able to bring out alone.

Le présent article a pour but de discuter la genèse et les limites de la notion de «limnicité » en lien avec les nouvelles problématiques sociales et sociétales, nées des incompréhensions multiples entre législateurs et acteurs de terrain autour des notions de masses d'eau "cours d'eau » et « plans d'eau » de la DCE.

Ces limites sont multiples et sont déjà visibles dans la construction même des outils d'aménagement des eaux actuels. En effet, dans le cadre de l'application de la DCE, les Agences de l'Eau prennent en compte les MEFM (masses d'eau fortement modifiées) en plus des lacs naturels alors qu'elles ne sont pas naturelles. Il s'agit donc d'une première transgression. Celle-ci se double néanmoins d'un ajout qui est le seuil surfacique : sans aucun argument limnique, celui-ci est fixé à 50 hectares, tout en offrant la latitude d'une interprétation tenant compte des réalités locales (abaissement du seuil, prise en compte de régions lacustres).

Ces choix ont deux conséquences: le critère de la seule naturalité lacustre est mis à mal et l'arbitraire surfacique entraîne une conflictualité sur le terrain entre acteurs de l'eau (moins de 1 $\%$ des plans d'eau français sont identifiés, le reste étant considéré par défaut comme faisant parti du cours d'eau) ; la notion même de superficie comme critère prioritaire est remis en question par une notion numérique propre aux régions lacustres.

Les auteurs proposent alors d'adapter la notion de «limnicité » à la complexité lentique et de prendre en compte la totalité des lacs, qu'ils soient naturels ou artificiels et, en parallèle, d'élargir l'éventail des plans d'eau étudiés en y intégrant les mares et les étangs qui, à l'échelle de la France, ont une superficie totale supérieure à celle des lacs (naturels et artificiels) : ainsi naît la notion de « limnicité étendue ».

Le choix de la dimension spatiale des plans d'eau comme critère dominant est ensuite débattu en lien avec les avancées scientifiques mettant en évidence la prégnance de la répétition du fait lacustre par rapport à l'impact d'une seule entité limnique, que ce soit pour les paramètres physico-chimiques de l'eau que pour le plan sociétal. De fait, la densité de plans d'eau est mise au même niveau d'analyse que la superficie de plans d'eau. Grâce à ce cheminement scientifique, deux nouveaux indicateurs permettant de mieux appréhender le rôle des plans d'eau dans le milieu sont élaborés : l'indicateur d'égale superficie (IES) et l'indicateur d'égale concentration (IEC). Appliqués à l'échelle départementale française, ces deux indicateurs font ressortir un fait majeur : les départements possédant les régions de plans d'eau les plus reconnues nationalement et internationalement et sur lesquels les modes de gestion actuels sont calés sont secondaires pour la compréhension des liens socio-environnementaux existant aujourd'hui au niveau des plans d'eau. Ce constat montre combien il existe de décalages entre la réalité de terrain des plans d'eau actuellement en France et ce qu'on voudrait qu'elle fût et que la seule notion de « limnicité » n'est pas à même de faire ressortir seule. 
Der vorliegende Artikel soll sowohl die Entstehung also auch die Grenzen des Begriffs Gewässeranteil diskutieren; dabei soll auf die neuen gesellschaftlichen und gesellschaftsstrukturellen Problemstellungen eingegangen werden, die sich aus der großen Zahl von Verständnisschwierigkeiten zwischen Gesetzgebung und ausführenden Personen oder Organen vor Ort vor dem Hintergrund der unterschiedlichen Interpretationen des Begriffs Gewässer (Gewässervolumen vs. Fließgewässer vs. stehendes Gewässer) in der Wasserrahmenrichtlinie ergeben haben.

Diese Grenzen zeigen sich an vielfacher Stelle und werden bereits im Aufbau der Werkzeuge offenbar, über welche die Wasserwirtschaftsämter verfügen. Denn im Rahmen der Umsetzung der Wasserrahmenrichtlinie beziehen die Wasserwirtschaftsämter neben den natürlichen Seen auch künstliche Gewässer (MEFM) mit ein, obwohl diese nicht-natürlichen Ursprungs sind. Hierbei handelt es sich demnach um eine erste Begriffsüberschreitung. $\mathrm{Zu}$ dieser tritt eine weitere hinzu, bei der es sich um den Schwellenwert hinsichtlich der Gewässeroberfläche handelt: ohne jegliche limnische Begründung wird dieser Wert auf 50 Hektar festgesetzt, wobei hier insofern (zusätzliche) Handlungsspielräume eröffnet werden, als bei der Auslegung ein Eingehen auf örtliche Gegebenheiten (Absenken des Grenzwerts, Berücksichtigung von Seengebieten) zulässig ist.

Diese (Wahl-)Entscheidungen führen zu zwei Konsequenzen: Zum einen wird das Kriterium der ausschließlichen Natürlichkeit der Gewässer gefährdet, zum anderen führt die Willkürlichkeit in der Festlegung der Gewässerfläche zu Konfliktsituationen vor Ort innerhalb der Wasserwirtschaftsorgane (weniger als $1 \%$ der stehenden Gewässer in Frankreich sind wasserrechtlich erfasst; der überwiegende Rest wird standardmäßig als Teil des Fließgewässers betrachtet). Der Begriff der Oberfläche selbst wird in seiner Rolle als vorrangiges Kriterium durch einen Zahlenwert infrage gestellt, der für das jeweilige Seengebiet gilt.

Die Autoren schlagen aus diesem Grund eine Anpassung des Begriffes Gewässeranteil an die Komplexität der Natur stehender Gewässer vor ebenso wie eine Berücksichtigung der Gesamtheit aller Seenflächen, seien sie nun natürlicher oder künstlicher Art. Parallel dazu soll das Spektrum der untersuchten Gewässer durch eine Einbeziehung der Teich- und Weiherflächen ausgeweitet werden, die auf das französische Staatsgebiet bezogen eine Gesamtfläche aufweisen, welche diejenige der Seen (natürlicher wie künstlicher Art) übersteigt: So entsteht der Begriff des Erweiterten Gewässeranteils.

Im Folgenden wird ein Ansatz der räumlichen Ausdehnung der Gewässer als vorrangiges Kriterium diskutiert; hierbei werden Verbindungen hergestellt zu den Forschungsansätzen, welche die Bedeutung der Wiederholung eines Einzelgewässers gegenüber dem Einfluss eines reinen Einzelgewässers hervorheben, sei es im Hinblick auf physisch-chemische Parameter des Wassers oder auch auf gesellschaftliche Einflussgrößen. Denn die Gewässerdichte wird ebenso detailliert untersucht wie die Gewässerfläche.

Dank dieser wissenschaftlichen Entwicklungen lassen sich zwei neue Indikatoren herausarbeiten, die eine bessere Erfassung der Rolle der Gewässer in ihrem natürlichen Umfeld erlauben: der Indikator gleicher Oberfläche sowie der Indikator gleicher Konzentration. Werden diese auf den Rahmen der französischen Départements bezogen, so lassen diese beiden Indikatoren ein bedeutsames Faktum klar hervortreten: die Départements, in denen die national wie international bedeutsamsten Seengebiete liegen und auf denen die die gegenwärtigen Verwaltungspraktiken beruhen, sind nur zweitrangig für das Verständnis derjenigen Zusammenhänge zwischen Gesellschaft und Umwelt, die heute im Bereich der Gewässer existieren. Dieser Befund verdeutlicht das gegenwärtige Ausmaß an Diskrepanzen, die in Frankreich im Bereich der Gewässer zwischen den Gegebenheiten vor Ort und den Wunschvorstellungen existieren; der bloße Begriff des Gewässeranteils ist nicht dazu geeignet, diese Diskrepanzen klar hervortreten zu lassen. 
INDEX

Schlüsselwörter: Gewässeranteil, Wasserfläche der Stillgewässer, weiter Gewässeranteil, Teich Weiher, See - [Wasser]tümpel, Gewässermorphometrie

Mots-clés: limnicité, stagnucité, limnicité étendue, morphométrie lacustre, étang, lac, mare.

Keywords: limnic ratio (after Meybeck) or limnicity (after Downing), stagnucity, extended limnic ratio, lake area, pond, lake, pool

\section{AUTHORS}

\section{PASCAL BARTOUT}

EA CEDETE 1210 - ITP HCS - Université d'Orléans. Collegium UFR LLSH. 10 rue de Tours 45036 Orléans. pascal.bartout@univ-orleans.fr

\section{LAURENT TOUCHART}

EA CEDETE 1210 - ITP HCS - Université d'Orléans. Collegium UFR LLSH. 10 rue de Tours 45036 Orléans. laurent.touchart@univ-orleans.fr 